\title{
Gastrointestinal Bleeding in Acute Ischemic Stroke: Recent Trends from the Fukuoka Stroke Registry
}

\author{
Toshiyasu Ogata a, b Masahiro Kamouchic, e Ryu Matsuo ${ }^{d}$ Jun Hatad, e \\ Junya Kurodad, e Tetsuro Ago ${ }^{d, e}$ Hiroshi Sugimori ${ }^{f}$ Tooru Inoue $^{b}$ \\ Takanari Kitazonod, e on behalf of the Fukuoka Stroke Registry \\ Departments of a Neurology and ${ }^{b}$ Neurosurgery, Faculty of Medicine, Fukuoka University, \\ Departments of ${ }^{c}$ Health Care Administration and Management and ${ }^{d}$ Medicine and Clinical \\ Science, Graduate School of Medical Sciences, Kyushu University, and e Department of \\ Nephrology, Hypertension, and Strokology and ${ }^{\mathrm{f} E m e r g e n c y ~ a n d ~ C r i t i c a l ~ C a r e ~ C e n t e r, ~}$ \\ Kyushu University Hospital, Fukuoka, Japan
}

Key Words

Ischemic stroke $\cdot$ Gastrointestinal bleeding · Outcome

\section{Abstract}

Background: Gastrointestinal (GI) hemorrhage is a potentially serious complication of acute stroke, but its incidence appears to be decreasing. The aim of this study was to elucidate the etiology of GI bleeding and its impact on clinical outcomes in patients with acute ischemic stroke in recent years. Methods: Using the database of the Fukuoka Stroke Registry, 6,529 patients with acute ischemic stroke registered between June 2007 and December 2012 were included in this study. We recorded clinical data including any previous history of peptic ulcer, prestroke drug history including the use of antiplatelets, anticoagulants, steroids and nonsteroidal anti-inflammatory drugs (NSAIDs), and poststroke treatment with suppressing gastric acidity. GI bleeding was defined as any episode of hematemesis or melena on admission or during hospitalization. The cause and origin of bleeding were diagnosed endoscopically. Logistic regression analysis was used to identify risk factors for GI bleeding and its influence on deteriorating neurologic function, death, and poor outcome. Results: GI bleeding occurred in 89 patients (1.4\%) under the condition that $66 \%$ of the total patients received acid-suppressing agents after admission. Multivariate analysis revealed that GI bleeding was associated with the absence of dyslipidemia ( $p=0.03)$, a previous history of peptic ulcer $(p<0.001)$, and the severity of baseline neurologic deficit ( $p=0.002)$ but not with antiplatelet drugs, anticoagulants, and NSAIDs. The source was the upper GI tract in $51 \%$ of the cases; causes in- 
cluded peptic ulceration (28\%) and malignancies (12\%), and other or unidentified causes accounted for $60 \%$. GI bleeding mostly occurred within 1 week after stroke onset. Hemoglobin concentration fell by a median value of $2.5 \mathrm{~g} / \mathrm{dl}$ in patients with GI bleeding. Among them, 28 patients underwent blood transfusion (31.5\%). After adjustment for confounding factors, GI bleeding was independently associated with neurologic deterioration (OR 3.9, 95\% CI 2.3-6.6, $p<0.001$ ), in-hospital death (OR 6.1,95\% CI 3.1-12.1, p < 0.001), and poor outcome at 3 months (OR 6.8, 95\% CI 3.7-12.7, $\mathrm{p}<0.001$ ). These associations were significant irrespective of whether patients underwent red blood cell transfusion. Conclusions: GI bleeding infrequently occurred in patients with acute ischemic stroke, which was mostly due to etiologies other than peptic ulcer. GI bleeding was associated with poor clinical outcomes including neurologic deterioration, in-hospital mortality, and poor functional outcome.

(c) 2014 S. Karger AG, Basel

\section{Introduction}

Gastrointestinal (GI) bleeding is a well-known complication that may occur during the acute phase of stroke [1-6] with an incidence of $0.1-8.0 \%$, depending on the population studied. Previous studies have identified various factors associated with GI bleeding after acute ischemic stroke $[1,3,4,6]$. One of the most common factors is a previous history of peptic ulcer [3]. However, suppressing gastric acidity by means of histamine-2 receptor antagonist (H2RA) or proton pump inhibitor (PPI) is an efficient means of preventing peptic ulcer. Moreover, the incidence of Helicobacter pylori infection has decreased owing to sanitary improvements and eradication therapy [7-9], and the prevalence of peptic ulcer has fallen in developed countries $[10,11]$. Accordingly, the incidence of upper GI bleeding has decreased in the general population [12].

Previous studies have reported the risk of GI bleeding during the acute phase of stroke in those who received little or no peptic ulcer prophylaxis. Recently, the use of prophylactic H2RAs or PPIs has become commonplace in the acute management of stroke in Japan, because it was recommended for elderly patients or those with severe stroke in the Japanese Guidelines for Management of Stroke published in 2004 or 2009 [13]. As these drugs reduce the risk of GI bleeding in patients on antithrombotic therapy $[14,15]$, the incidence, etiology, and clinical significance of GI bleeding as a complication of stroke may have changed. However, the current data concerning GI bleeding in acute stroke are limited.

The purpose of this study was to clarify the current incidence of GI bleeding and its clinical significance in patients with acute ischemic stroke. For this purpose, we investigated the incidence and endoscopic diagnosis of GI bleeding, the factors associated with GI bleeding, and the association of GI bleeding with clinical outcomes in a large cohort of patients with acute ischemic stroke.

\section{Methods}

\section{Subject Recruitment}

The Fukuoka Stroke Registry (FSR) is a multicenter, hospital-based registry in which acute stroke patients are enrolled [16-18]. The Kyushu University Hospital and six other stroke centers in Fukuoka, Japan, participate in this registry (see Appendix). The institutional review board approved the study protocols. We received written informed consent from all patients or their proxies. All patients who had a stroke and who were hospitalized within 7 days of symptom onset in participating hospitals were prospectively registered in the FSR. 
Stroke was defined as a sudden onset of nonconvulsive and focal neurologic deficit. Ischemic stroke was confirmed by means of brain imaging, including computed tomography and/or magnetic resonance imaging. We included all patients with ischemic stroke or transient ischemic attack hospitalized within 7 days of onset between June 2007 and December 2012 and excluded those with hemorrhagic stroke. We retrospectively reviewed this dataset. Through the use of TOAST criteria [19], we further subclassified ischemic stroke into cardioembolic and noncardioembolic subtypes (i.e. small vessel, large vessel, and unclassified). Of the 7,127 patients consecutively registered, 598 patients with hemorrhagic stroke were excluded. The data of 6,529 patients were examined.

\section{Clinical Data}

We recorded age, gender, prestroke modified Rankin Scale, body mass index, and risk factors. Hypertension was defined as systolic blood pressure $\geq 140 \mathrm{~mm} \mathrm{Hg}$ or diastolic blood pressure $\geq 90 \mathrm{~mm} \mathrm{Hg}$ in the chronic stage or a history of treatment with antihypertensive drugs. Dyslipidemia was defined as a serum low-density lipoprotein level $\geq 3.62 \mathrm{mmol} / \mathrm{l}$, a high-density lipoprotein level $<1.03 \mathrm{mmol} / \mathrm{l}$, triglycerides $\geq 1.69 \mathrm{mmol} / \mathrm{l}$, or a history of treatment with a cholesterol-lowering drug. Atrial fibrillation was diagnosed based on the electrocardiographic findings on admission and during hospitalization or a previous history of atrial fibrillation. Smoking was defined as a current or a former habit of cigarette smoking, and alcohol intake as habitual consumption of alcohol beverages before onset of stroke.

We also recorded any previous history of stroke or peptic ulcer, prestroke drug history including the use of antiplatelets, anticoagulants, steroids, and nonsteroidal anti-inflammatory drugs (NSAIDs), and poststroke treatment with H2RAs or PPIs, thrombolytic therapy, antiplatelets, anticoagulants, and statins. Thrombolytic treatment was defined as intravenous or intra-arterial administration of thrombolytic agents such as recombinant tissue-type plasminogen activator and urokinase in the acute phase of stroke.

\section{Definition of GI Bleeding}

GI bleeding was defined as any episode of hematemesis or melena during hospitalization $[4,6]$. The cause and origin of the bleeding were investigated using endoscopy in all cases.

\section{Clinical Outcomes}

Neurological severity was assessed by the National Institute of Health Stroke Scale (NIHSS). Neurological deterioration was defined as an increase in the NIHSS score of 4 points or more during hospitalization. In-hospital mortality was defined as all causes of death during hospitalization. Functional outcome was evaluated with a telephone interview 3 months after symptom onset, and a modified Rankin Scale $\geq 3$ was defined as poor functional outcome. As the Rankin Scale data were not available for 47 patients, these patients were excluded from the functional outcome analysis.

\section{Statistical Analyses}

Univariate analysis was undertaken using the unpaired t test, Mann-Whitney U test, and $\chi^{2}$ test after the distribution of the data had been examined using the Shapiro-Wilk test. The factors associated with GI bleeding were investigated using univariate and multivariate analyses. The multivariate model included the variables found to have a p value $<0.05$. To examine whether GI bleeding was associated with deteriorating neurologic function, death, and poor outcome, we adjusted for multiple confounding factors including age, gender, stroke subtype, systolic blood pressure on admission, hypertension, atrial fibrillation, smoking history, serum glucose, NIHSS on admission, and thrombolytic treatment. Data were analyzed using SPSS version 21.0 (IBM SPSS, Somers, N.Y., USA). 

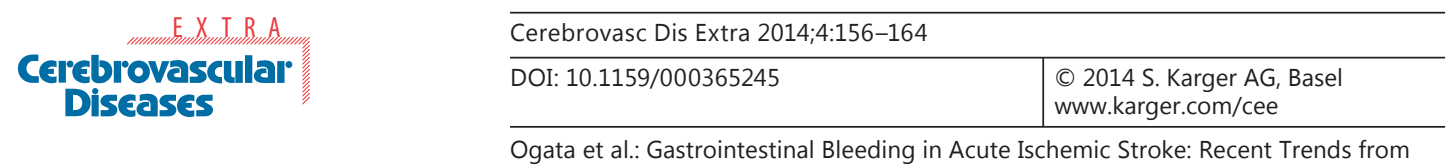

Ogata et al.: Gastrointestinal Bleeding in Acute Ischemic Stroke: Recent Trends from the Fukuoka Stroke Registry

Table 1. Background characteristics of patients with and without GI bleeding

\begin{tabular}{|c|c|c|c|}
\hline & $\begin{array}{l}\text { GI bleeding } \\
(\mathrm{n}=89)\end{array}$ & $\begin{array}{l}\text { No GI bleeding } \\
(n=6,440)\end{array}$ & $\mathrm{p}$ \\
\hline Age, years & $76(70-83)$ & $73(64-81)$ & 0.01 \\
\hline Male & $53(59.6)$ & 3,845 (59.7) & 1.00 \\
\hline Prestroke mRS score & $0(0-2)$ & $0(0-1)$ & 0.046 \\
\hline Body mass index & $21.9(19.2-24.8)$ & $22.7(20.4-25.1)$ & 0.07 \\
\hline \multicolumn{4}{|l|}{ Risk factors } \\
\hline Hypertension & $68(76.4)$ & $5,059(78.6)$ & 0.60 \\
\hline Dyslipidemia & $26(29.2)$ & $3,197(49.6)$ & $<0.001$ \\
\hline Diabetes mellitus & $31(34.8)$ & $1,975(30.7)$ & 0.42 \\
\hline Atrial fibrillation & $32(36.0)$ & $1,613(25.0)$ & 0.03 \\
\hline Current smoker & $45(50.6)$ & $3,264(50.7)$ & 1.00 \\
\hline Current alcohol drinker & $33(37.1)$ & $2,234(34.7)$ & 0.66 \\
\hline \multicolumn{4}{|l|}{ Previous history } \\
\hline Stroke & $21(23.6)$ & 1,397 (21.7) & 0.70 \\
\hline Peptic ulcer & $27(30.3)$ & $379(5.9)$ & $<0.001$ \\
\hline \multicolumn{4}{|l|}{ Stroke subtypes } \\
\hline Cardioembolic & $30(33.7)$ & $1,435(22.3)$ & 0.01 \\
\hline Baseline NIHSS score & $6(3-13)$ & $3(1-6)$ & $<0.001$ \\
\hline \multicolumn{4}{|l|}{ Prestroke treatment } \\
\hline Antiplatelet & $28(31.5)$ & 1,917 (29.8) & 0.73 \\
\hline Anticoagulant & $12(13.5)$ & $618(9.6)$ & 0.21 \\
\hline Steroid & $5(5.6)$ & $141(2.2)$ & 0.049 \\
\hline NSAIDs & $4(4.5)$ & $266(4.1)$ & 0.79 \\
\hline Statin & $23(25.8)$ & $2,483(38.6)$ & 0.02 \\
\hline \multicolumn{4}{|l|}{ Poststroke treatment } \\
\hline Thrombolytic agent & $9(10.1)$ & $454(7.0)$ & 0.29 \\
\hline Antiplatelet & $59(66.1)$ & $5,039(78.2)$ & 0.01 \\
\hline Anticoagulant & $74(83.1)$ & $4,752(73.8)$ & 0.051 \\
\hline H2RA or PPI & $60(67.4)$ & $4,226(65.6)$ & 0.82 \\
\hline
\end{tabular}

Values are median (interquartile range) or $\mathrm{n}(\%)$. mRS = Modified Rankin Scale.

\section{Results}

\section{Baseline Characteristics and GI Bleeding}

GI bleeding occurred in 89 patients during hospitalization (1.4\%). Baseline characteristics of the patients with and without GI bleeding are shown in table 1. An H2RA or PPI was administered to 4,286 patients (65.6\%) after admission. Those who experienced GI bleeding were older, and their activities of daily living were more impaired before onset. Dyslipidemia was less frequent, but atrial fibrillation, a history of peptic ulcer, and cardioembolic stroke were more prevalent in patients with GI bleeding compared with those without. The NIHSS score on admission was significantly higher in those with GI bleeding. Both groups had a comparable frequency of prestroke treatment with antiplatelet drugs, anticoagulants, and NSAIDs, although steroid use before stroke onset was more frequent in patients with GI bleeding. Regarding poststroke treatment, antiplatelets and statins were less frequently administered in patients with GI bleeding.

After adjusting for potential confounding factors, the absence of dyslipidemia, a history of peptic ulcer, and an NIHSS score on admission were identified to be significant factors associated with the occurrence of GI bleeding (table 2). Prestroke use of steroid was marginally 


\section{Cerebrovascular \\ Diseases}

Table 2. Factors associated with GI bleeding

Table 3. Origin and cause of GI bleeding

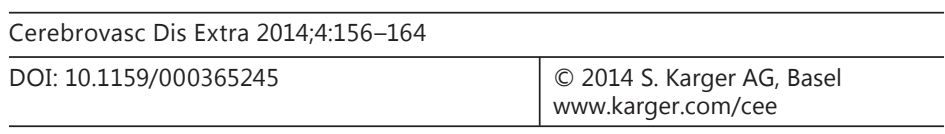

Ogata et al.: Gastrointestinal Bleeding in Acute Ischemic Stroke: Recent Trends from the Fukuoka Stroke Registry

\begin{tabular}{lllc}
\hline & OR & $95 \%$ CI & p \\
\hline Dyslipidemia & 0.50 & $0.27-0.93$ & 0.03 \\
Previous peptic ulcer & 6.59 & $4.11-10.57$ & $<0.001$ \\
Baseline NIHSS score (per 1 score) & 1.05 & $1.02-1.08$ & 0.002 \\
Prestroke treatment with steroid & 2.49 & $0.96-6.44$ & 0.06 \\
\hline
\end{tabular}

The multivariate model included age, prestroke modified Rankin Scale score, dyslipidemia, atrial fibrillation, previous peptic ulcer, cardioembolic stroke, baseline NIHSS score, prestroke steroid, prestroke statin, and poststroke antiplatelet. Baseline NIHSS was assessed on admission.

\begin{tabular}{lrl}
\hline Origin of bleeding & & \\
Esophagus & $12(13.4)$ & \\
Stomach & $22(24.7)$ & \\
Duodenum & $11(12.3)$ & \\
Colon & $14(15.7)$ & \\
Rectum & $9(10.1)$ & \\
Unknown & $21(23.6)$ & \\
\hline Cause of bleeding & & \\
Ulcer & & \\
$\quad$ Peptic ulcer & $25(28.1)$ & S 16, D 9 \\
$\quad$ Others & $9(10.1)$ & E 3, C 1, R 5 \\
Cancer & $11(12.3)$ & E 1, S 4, C 5, R 1 \\
Others & & \\
$\quad$ Erosion & $5(5.6)$ & E 1, S 2, D 1, R 1 \\
$\quad$ Polyp & $5(5.6)$ & C 3, R 2 \\
$\quad$ Reflux esophagitis & $4(4.5)$ & \\
$\quad$ Pseudomembranous enterocolitis & $2(2.2)$ & \\
$\quad$ Mallory-Weiss syndrome & $2(2.2)$ & \\
Esophageal varix & $1(1.1)$ & \\
Ischemic colitis & $1(1.1)$ & \\
Colon diverticulum & $1(1.1)$ & \\
Angiodysplasia of the colon & $1(1.1)$ & \\
Bleeding at duodenum due to & & \\
invasion of pancreatic cancer & $1(1.1)$ & \\
$\quad$ Unidentified & $21(23.6)$ & \\
\hline
\end{tabular}

Values are $\mathrm{n}(\%)$. The localization of bleeding is shown as follows: $\mathrm{E}=$ esophagus; $\mathrm{S}=$ stomach; $\mathrm{D}=$ duodenum; $\mathrm{C}=$ colon; $\mathrm{R}=$ rectum.

associated with GI bleeding, whereas no association was found between the other factors and GI bleeding ( $p$ values $\geq 0.10$, data not shown).

\section{Etiology of GI Bleeding}

Endoscopic examination revealed that the bleeding originated in both the upper and lower GI tracts (table 3). In $23.6 \%$ of the patients, the origin of GI bleeding could not be identified despite endoscopic investigation. Although peptic ulcer was a common cause of the bleeding, others were also observed. In 25 patients in whom GI bleeding was caused by peptic 

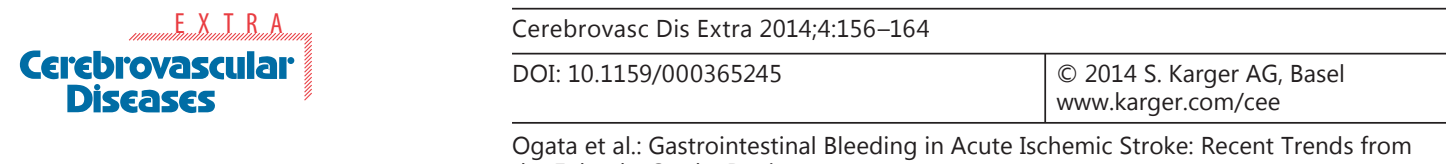

Ogata et al.: Gastrointestinal Bleeding in Acute Ischemic Stroke: Recent Trends from the Fukuoka Stroke Registry

Table 4. Association between GI bleeding with or without blood transfusion and clinical outcomes

\begin{tabular}{|c|c|c|c|c|c|c|c|}
\hline & \multirow{2}{*}{$\begin{array}{l}\text { Number of } \\
\text { events (\%) }\end{array}$} & \multicolumn{3}{|c|}{ Age- and gender-adjusted } & \multicolumn{3}{|c|}{ Multivariate-adjusted } \\
\hline & & OR & $95 \% \mathrm{CI}$ & $\mathrm{p}$ & OR & $95 \% \mathrm{CI}$ & $\mathrm{p}$ \\
\hline \multicolumn{8}{|l|}{ Neurologic deterioration } \\
\hline Non-GI bleeding & $391 / 6,440(6.1)$ & 1.00 & reference & & 1.00 & reference & \\
\hline \multicolumn{8}{|l|}{ GI bleeding } \\
\hline Overall & $22 / 89(24.7)$ & 4.74 & $2.88-7.80$ & $<0.001$ & 3.87 & $2.27-6.61$ & $<0.001$ \\
\hline Without transfusion & $13 / 61(21.3)$ & 3.90 & $2.08-7.32$ & 0.001 & 3.17 & $1.63-6.17$ & 0.001 \\
\hline With transfusion & $9 / 28(32.1)$ & 6.85 & $3.06-15.35$ & $<0.001$ & 5.77 & $2.38-14.02$ & $<0.001$ \\
\hline \multicolumn{8}{|l|}{ In-hospital death } \\
\hline $\begin{array}{l}\text { Non-GI bleeding } \\
\text { GI bleeding }\end{array}$ & $124 / 6,440$ (1.9) & 1.00 & reference & & 1.00 & reference & \\
\hline Overall & $14 / 89$ (15.7) & 8.69 & $4.71-16.05$ & $<0.001$ & 6.08 & $3.06-12.11$ & $<0.001$ \\
\hline Without transfusion & $10 / 61(16.4)$ & 9.05 & $4.39-18.66$ & $<0.001$ & 5.89 & $2.68-12.97$ & $<0.001$ \\
\hline With transfusion & $4 / 28(14.3)$ & 8.02 & $2.68-23.97$ & $<0.001$ & 6.72 & $1.77-25.54$ & 0.005 \\
\hline \multicolumn{8}{|l|}{ Poor functional outcome } \\
\hline Non-GI bleeding & 2,177/6,395 (34.0) & 1.00 & reference & & 1.00 & reference & \\
\hline \multicolumn{8}{|l|}{ GI bleeding } \\
\hline Overall & 68/87 (78.2) & 7.95 & $4.58-13.80$ & $<0.001$ & 6.82 & $3.67-12.68$ & $<0.001$ \\
\hline Without transfusion & $46 / 59(78.0)$ & 8.23 & $4.19-16.16$ & $<0.001$ & 6.87 & $3.13-15.07$ & $<0.001$ \\
\hline With transfusion & $22 / 28(78.6)$ & 7.42 & $2.87-19.22$ & $<0.001$ & 6.77 & $2.49-18.44$ & $<0.001$ \\
\hline
\end{tabular}

The multivariate model included age, gender, stroke subtype, systolic blood pressure on admission, hypertension, atrial fibrillation, smoking habit, serum glucose on admission, NIHSS score on admission, and thrombolytic treatment.

ulcer, 23 patients had a previous history of peptic ulcer. Among them, 18 patients $(78.3 \%)$ were treated with H2RA (4 patients) or PPI (14 patients), whereas 5 patients were treated with neither of them.

\section{GI Bleeding and Clinical Outcomes}

GI bleeding mostly occurred within 1 week after stroke onset (median 4 days, interquartile range 1-16 days). Hemoglobin concentration fell by a median value of $2.5 \mathrm{~g} / \mathrm{dl}$ (interquartile range 1.8-4.0) in patients with GI bleeding. Among them, 28 patients underwent blood transfusion (31.5\%). The frequency of neurologic deterioration during hospitalization and poor functional outcome at 3 months was higher in patients with GI bleeding than in those without. All-cause mortality during hospitalization was also higher in patients with GI bleeding. After adjustment for confounding factors, GI bleeding was independently associated with poor clinical outcomes including neurologic deterioration, in-hospital mortality, and poor functional outcome at 3 months (table 4). Additionally, these associations were significant irrespective of whether patients underwent red blood cell transfusion (table 4).

\section{Discussion}

We found that the incidence of GI bleeding during hospitalization was $1.4 \%$ in patients with acute ischemic stroke in the past 5 years. A previous retrospective study conducted in Asia (January 2001 to October 2005) using the same definition of GI bleeding reported a 
frequency of 7.8\% [6]. In that study, only 5\% of the patients received H2RA or PPI, and endoscopic examination showed that GI bleeding was caused by peptic ulcer in $74 \%$ of the patients who underwent endoscopy [6]. Another study using the Registry of the Canadian Stroke Network (July 2003 to June 2006) showed that GI bleeding was relatively uncommon after acute ischemic stroke, with an incidence of 1.5\% [1]. In that study, H2RA or PPI had been administered to $21 \%$ of the patients before admission, but the frequency of the post-admission treatment and the etiology of GI bleeding were not reported. In our study, approximately two thirds of the patients were treated with H2RA or PPI, and 72\% of the bleeding events were due to pathologies other than peptic ulcer. The site of bleeding was almost equally distributed between the lower and upper GI tract. Therefore, during recent years, GI bleeding occurs from the lower as well as the upper GI tract and from other sources such as malignancies, erosions, and polyps in Japanese patients with acute ischemic stroke.

We investigated whether factors associated with GI bleeding in this cohort were the same as those reported previously. In this study, the severity of neurologic impairment was significantly associated with GI bleeding, which concurs with the findings of other studies $[1,4]$. Furthermore, a history of peptic ulcer is still associated with an increased risk of GI bleeding. The multivariate-adjusted OR for GI bleeding was 6.6 in patients with a history of peptic ulcer, suggesting that GI bleeding can develop in those with previous peptic ulcer despite acidsuppressing drugs. In contrast, the use of antithrombotic agents or NSAIDs was not associated with GI bleeding, which contradicts previous findings [3]. Additionally, the association of prestroke steroid use with GI bleeding was statistically marginal after adjustment for confounders in our study. The adverse effects of these drugs on GI bleeding may have been attenuated compared with the past. In this study, dyslipidemia was found to be associated with a reduced risk of GI bleeding. However, we are unable to suggest an underlying mechanism, and there may be an unidentified confounding factor. Previous studies showed that the eradication of $H$. pylori is associated with the future elevation of the serum levels of cholesterol or triglyceride and hence dyslipidemia [20-22]. Such an association may underlie a reduced risk for GI bleeding in patients with dyslipidemia. Further studies are needed to confirm this potential association in more detail.

This study showed that GI bleeding was associated with poor clinical outcomes including neurologic deterioration, in-hospital mortality, and poor functional outcome. GI bleeding remains a substantial and significant influence on the clinical outcomes of patients who suffered an acute ischemic stroke. Previous studies have shown that GI bleeding was associated with death or functional dependency $[1,4]$, but its relationship with the neurological course is less clear. In this study, GI bleeding was also associated with neurologic deterioration. There are some possibilities regarding the mechanisms for the association between GI bleeding and poor clinical outcomes. Bleeding does not only result in hemodynamic insufficiency but also, importantly, in the discontinuation of antithrombotic treatment that leads to a prothrombotic state [23]. In this study, the risks of poor clinical outcomes were significantly higher in patients with GI bleeding irrespective of whether they required transfusion of red blood cells for anemia. Although the causation between GI bleeding and poor clinical outcomes cannot be proven from this study design, cessation of antithrombotic therapy rather than hemodynamic instability may lead to the deterioration of neurological symptoms and poor functional outcome. Further study is required to elucidate the mechanisms for their association.

Our study has some limitations. We could not detect all patients with GI bleeding because those who showed a gradual decrease in hemoglobin without hematemesis or melena were not included. In addition, the cause of GI bleeding was not identified in $23.6 \%$ of the patients despite endoscopic examination. Endoscopic examination may not have detected the origin of GI bleeding because the GI tract could not be thoroughly examined in an emergency. Treat- 
ments performed before and after stroke onset were not controlled but determined by each attending doctor, which led to confounding by indication. The onset of GI bleeding was ambiguous because we defined it with episodes of hematemesis or melena, leading to an uncertain relationship between GI bleeding and the administration of antithrombotics or acid-suppressing agents. Validation of our findings is required in other cohorts of different ethnic groups to further elucidate the incidence and clinical significance of GI bleeding in patients with acute ischemic stroke.

\section{Appendix}

\section{FSR Investigators}

The participating hospitals in the FSR included the Kyushu University Hospital, National Hospital Organization Kyushu Medical Center, National Hospital Organization Fukuoka Higashi Medical Center, Fukuoka Red Cross Hospital, St. Mary's Hospital, Steel Memorial Yawata Hospital, and Japan Labour Health and Welfare Organization Kyushu Rosai Hospital.

The Steering Committee included Takao Ishitsuka, MD (Steel Memorial Yawata Hospital); Shigeru Fujimoto, MD (Steel Memorial Yawata Hospital); Setsuro Ibayashi, MD (Seiai Rehabilitation Hospital); Kenji Kusuda, MD (Seiai Rehabilitation Hospital); Shuji Arakawa, MD (Japan Labour Health and Welfare Organization Kyushu Rosai Hospital); Katsumi Irie, MD (Hakujyuji Hospital); Kenichiro Fujii, MD (Fukuoka Red Cross Hospital); Yasushi Okada, MD (National Hospital Organization Kyushu Medical Center); Masahiro Yasaka, (National Hospital Organization Kyushu Medical Center); Tetsuhiko Nagao, MD (Midrino Clinic); Hiroaki Ooboshi, MD (Fukuoka Dental Collage Medical and Dental Hospital); Tsuyoshi Omae, MD (Imazu Red Cross Hospital); Kazunori Toyoda, MD (National Cerebral and Cardiovascular Center); Hiroshi Nakane, MD (National Hospital Organization Fukuoka-Higashi Medical Center); Kenji Fukuda, MD (St. Mary's Hospital); Yoshihisa Fukushima, MD (St. Mary's Hospital); Kinya Tamaki, MD (Shinyoshizuka Hospital); Seizo Sadoshima, MD (Shinyoshizuka Hospital).

\section{Acknowledgments}

This study was supported by the JSPS KAKENHI grant Nos. 22249069, 24310024, and 26293158 and by the Coordination, Support and Training Program for Translational Research from the Japanese Ministry of Education, Culture, Sports, Science and Technology. We thank Assoc. Prof. Hitoshi Inoue (Research Institute for Information Technology, Kyushu University) for his technical support with the secure FSR Data Collection System and all clinical research coordinators (Hisayama Research Institute for Lifestyle Diseases) for their help in obtaining informed consent and collecting clinical data.

\section{Disclosure Statement}

The authors have no conflicts of interest to disclose. 


\section{Cerebrovascular Diseases}

Ogata et al.: Gastrointestinal Bleeding in Acute Ischemic Stroke: Recent Trends from the Fukuoka Stroke Registry

\section{References}

1 O’Donnell MJ, Kapral MK, Fang J, Saposnik G, Eikelboom JW, Oczkowski W, Silva J, Gould L, D'Uva C, Silver FL: Gastrointestinal bleeding after acute ischemic stroke. Neurology 2008;71:650-655.

-2 Johnston KC, Li JY, Lyden PD, Hanson SK, Feasby TE, Adams RJ, Faught RE Jr, Haley EC Jr: Medical and neurological complications of ischemic stroke: experience from the RANTTAS trial. RANTTAS Investigators. Stroke 1998;29:447-453.

-3 Wijdicks EF, Fulgham JR, Batts KP: Gastrointestinal bleeding in stroke. Stroke 1994;25:2146-2148.

4 Davenport RJ, Dennis MS, Warlow CP: Gastrointestinal hemorrhage after acute stroke. Stroke 1996;27:421424.

5 Misra UK, Kalita J, Pandey S, Mandal SK: Predictors of gastrointestinal bleeding in acute intracerebral haemorrhage. J Neurol Sci 2003;208:25-29.

6 Hsu HL, Lin YH, Huang YC, Weng HH, Lee M, Huang WY, Lee JD: Gastrointestinal hemorrhage after acute ischemic stroke and its risk factors in Asians. Eur Neurol 2009;62:212-218.

-7 Roosendaal R, Kuipers EJ, Buitenwerf J, van Uffelen C, Meuwissen SG, van Kamp GJ, Vandenbroucke-Grauls CM: Helicobacter pylori and the birth cohort effect: evidence of a continuous decrease of infection rates in childhood. Am J Gastroenterol 1997;92:1480-1482.

-8 Groenen MJ, Kuipers EJ, Hansen BE, Ouwendijk RJ: Incidence of duodenal ulcers and gastric ulcers in a western population: back to where it started. Can J Gastroenterol 2009;23:604-608.

-9 Fujisawa T, Kumagai T, Akamatsu T, Kiyosawa K, Matsunaga Y: Changes in seroepidemiological pattern of helicobacter pylori and hepatitis a virus over the last 20 years in Japan. Am J Gastroenterol 1999;94:20942099.

10 Sonnenberg A: Causes underlying the birth-cohort phenomenon of peptic ulcer: analysis of mortality data 1911-2000, England and Wales. Int J Epidemiol 2006;35:1090-1097.

11 Sonnenberg A: Differences in the birth-cohort patterns of gastric cancer and peptic ulcer. Gut 2010;59:736743.

12 Lanas A, Garcia-Rodriguez LA, Polo-Tomas M, Ponce M, Alonso-Abreu I, Perez-Aisa MA, Perez-Gisbert J, Bujanda L, Castro M, Munoz M, Rodrigo L, Calvet X, Del-Pino D, Garcia S: Time trends and impact of upper and lower gastrointestinal bleeding and perforation in clinical practice. Am J Gastroenterol 2009;104:1633-1641.

-13 Shinohara Y, Yanagihara T, Abe K, Yoshimine T, Fujinaka T, Chuma T, Ochi F, Nagayama M, Ogawa A, Suzuki N, Katayama Y, Kimura A, Kobayashi S: I. Stroke in general. J Stroke Cerebrovasc Dis 2011;20:S7-S30.

14 Lai KC, Lam SK, Chu KM, Wong BC, Hui WM, Hu WH, Lau GK, Wong WM, Yuen MF, Chan AO, Lai CL, Wong J: Lansoprazole for the prevention of recurrences of ulcer complications from long-term low-dose aspirin use. N Engl J Med 2002;346:2033-2038.

$\checkmark 15$ Lin KJ, Hernandez-Diaz S, Garcia Rodriguez LA: Acid suppressants reduce risk of gastrointestinal bleeding in patients on antithrombotic or anti-inflammatory therapy. Gastroenterology 2011;141:71-79.

16 Kamouchi M, Matsuki T, Hata J, Kuwashiro T, Ago T, Sambongi Y, Fukushima Y, Sugimori H, Kitazono T: Prestroke glycemic control is associated with the functional outcome in acute ischemic stroke: the Fukuoka stroke registry. Stroke 2011;42:2788-2794.

17 Kamouchi M, Kumagai N, Okada Y, Origasa H, Yamaguchi T, Kitazono T: Risk score for predicting recurrence in patients with ischemic stroke: the Fukuoka stroke risk score for Japanese. Cerebrovasc Dis 2012;34:351357.

18 Kuwashiro T, Sugimori H, Ago T, Kamouchi M, Kitazono T; FSR Investigators: Risk factors predisposing to stroke recurrence within one year of non-cardioembolic stroke onset: the Fukuoka Stroke Registry. Cerebrovasc Dis 2012;33:141-149.

19 Adams HP Jr, Bendixen BH, Kappelle LJ, Biller J, Love BB, Gordon DL, Marsh EE 3rd: Classification of subtype of acute ischemic stroke. Definitions for use in a multicenter clinical trial. TOAST. Trial of Org 10172 in Acute Stroke Treatment. Stroke 1993;24:35-41.

20 Papamichael KX, Papaioannou G, Karga H, Roussos A, Mantzaris GJ: Helicobacter pylori infection and endocrine disorders: is there a link? World J Gastroenterol 2009;15:2701-2707.

21 Kamada T, Hata J, Kusunoki H, Ito M, Tanaka S, Kawamura Y, Chayama K, Haruma K: Eradication of helicobacter pylori increases the incidence of hyperlipidaemia and obesity in peptic ulcer patients. Dig Liver Dis 2005;37:39-43.

-22 Fujiwara Y, Higuchi K, Arafa UA, Uchida T, Tominaga K, Watanabe T, Arakawa T: Long-term effect of Helicobacter pylori eradication on quality of life, body mass index, and newly developed diseases in Japanese patients with peptic ulcer disease. Hepatogastroenterology 2002;49:1298-1302.

23 Eikelboom JW, Mehta SR, Anand SS, Xie C, Fox KA, Yusuf S: Adverse impact of bleeding on prognosis in patients with acute coronary syndromes. Circulation 2006;114:774-782. 